\title{
Radio Frequency Interference
}




\title{
RADIO SPECTRUM MANAGEMENT AND RFI IN THE UNITED STATES
}

\author{
R. MARCUS PRICE \\ University of New Mexico, Albuquerque, NM U.S.A.
}

\begin{abstract}
In the United States, civil common carrier telecommunications are provided by private companies, not by any agency of the government. Regulation of these services and spectrum management oversight is provided by the Federal Communications Commission (FCC), an agency of the government. Government telecommunications are operated by individual agencies, e.g. the Department of Defense, under the overall regulation of the Office of Spectrum Management of the National Telecommunications and Information Administration (NTIA), a government body separate from the FCC. In bands shared by the civil and government sectors, liaison and coordination is effected between the FCC and the NTIA.
\end{abstract}

The use of the spectrum for scientific research in both the civil and government bands is carried out by private and government organizations. Research scientists have input to both the FCC and the NTIA. The Committee on Radio Frequencies (CORF), a standing committee of the National Academy of Sciences, provides comments and expert advice in response to various FCC actions and rulemaking procedures. The CORF also communicates its concerns to the Electromagnetic Spectrum Manager of the National Science Foundation (NSF), the major government funding source of basic research in the United States. The NSF Electromagnetic Spectrum Manager has access to the NTIA policy apparatus through the membership of the NSF in the Interdepartment Radio Advisory Committee (IRAC). The IRAC is the policy board for the government agencies in telecommunications matters.

Thus, individual scientists with concerns about frequency selection or RFI can communicate either with the CORF or the Electromagnetic Spectrum Manager of the NSF. This has been a successful, if somewhat complex, way to insure continued access to the spectrum for scientists.

Good spectrum management has, and coordination has, minimized the effects of RFI on scientific research uses of the spectrum. The major difficulties are in bands close to high powered radars, satellite down links, and extensive mobile operations. Especially in the bands from 1300 to 1400 $\mathrm{MHz}$ and near the $\mathrm{OH}$ lines, satellite systems have caused extensive interference. The rising use of the spectrum for portable devices, remote control applications, and security purposes, will result in a proliferation of hardware with the potential for interference to passive research programs. Finally, the use of unlicensed devices giving off incidental, spurious, and harmonic emissions is increasing. This rising tide of radio noise will make research using passive techniques increasingly difficult. 